\title{
BMJ Open Preventive effect of oral magnesium in postmastectomy pain: protocol for a randomised, double-blind, controlled clinical trial
}

\author{
Véronique Morel, ${ }^{1}$ Dominique Joly, ${ }^{2}$ Christine Villatte, ${ }^{2}$ Bruno Pereira, ${ }^{3}$ \\ Gisèle Pickering ${ }^{1,4,5}$
}

To cite: Morel V, Joly D, Villatte $\mathrm{C}$, et al. Preventive effect of oral magnesium in postmastectomy pain: protocol for a randomised, double-blind, controlled clinical trial. BMJ Open 2018;8:e017986. doi:10.1136/ bmjopen-2017-017986

- Prepublication history for this paper is available online. To view these files, please visit the journal online (http://dx.doi. org/10.1136/bmjopen-2017017986).

Received 20 September 2017 Revised 8 March 2018 Accepted 23 May 2018

\section{Check for updates}

(c) Author(s) (or their employer(s)) 2018. Re-use permitted under CC BY-NC. No commercial re-use. See rights and permissions. Published by BMJ.

For numbered affiliations see end of article.

Correspondence to Dr Véronique Morel; v_morel@chu-clermontferrand. $\mathrm{fr}$

\section{ABSTRACT}

Introduction Breast cancer affects 1 in 10 women worldwide, and mastectomy is a cause of chronic pain with neuropathic characteristics. $\mathrm{N}$-methyl-D-aspartate receptor (NMDAR) antagonists such as ketamine, memantine, dextromethorphan or magnesium are used to treat refractory pain by blocking NMDAR. Oral memantine has been shown to prevent postmastectomy pain and cognitive impact and to maintain quality of life. Likewise, the present study is intended to assess the preventive effect of oral magnesium, administered ahead of mastectomy, on the development of neuropathic pain. As a physiological blocker of NMDAR, magnesium could be an interesting candidate to prevent postoperative pain and associated comorbidities, including cognitive and emotional disorders, multiple analgesic consumption and impaired quality of life.

Methods and analysis A randomised double-blind controlled clinical trial (NCT03063931) will include 100 women with breast cancer undergoing mastectomy at the Oncology Hospital, Clermont-Ferrand, France. Magnesium (100 mg/day; $n=50)$ or placebo $(n=50)$ will be administered for 6 weeks, starting 2 weeks before surgery. Intensity of pain, cognitive and emotional function and quality of life will be assessed by questionnaires. The primary endpoint is pain intensity on a $0-10$ numerical rating scale at 1 month postmastectomy. Data analysis will use mixed models; all tests will be two-tailed, with type-I error set at $\alpha=0.05$.

Ethics and dissemination The study protocol and informed consent form were approved in December 2016 by the French Research Ethics Committee (South East VI Committee). Results will be communicated in various congresses and published in international publications. Trial registration number NCT03063931.

\section{INTRODUCTION}

Breast cancer is the most common cancer in women worldwide, and the lifetime probability of developing breast cancer is $12.3 \%$, or approximately 1 in $8 .{ }^{1}$ Mastectomy, chemotherapy and radiotherapy are important contributors to the development of neuropathic pain. ${ }^{2}$ Neuropathic pain is defined as pain related to a lesion or disease affecting the somatosensory system. The mechanisms responsible for spinal hyperexcitability include the activation of central and peripheral $N$-methyl-D-aspartate receptors (NMDAR) ${ }^{34}$ which play an ubiquitous role in central pain sensitisation and in many other functions, including memory and learning. ${ }^{5-7}$ Neuropathic pain is associated with the development of a number of comorbidities, including cognitive-emotional and sleep disorders. ${ }^{8}$ Postmastectomy pain may be reported in the anterior thorax, armpit or upper arm, and oedema, sensory dysfunction, neuroma and numbness in the arm contribute to the pain syndrome that affects $20 \%-68 \%$ of the patients. ${ }^{2}$ NMDAR antagonists such as ketamine, memantine, dextromethorphan or magnesium may, by blocking NMDAR, limit or even reverse the painful phenomena and are possible drugs for pain refractory to classical treatments. ${ }^{59-11}$

On a translational approach, the prophylactic effect of memantine in neuropathic pain was recently demonstrated in animals and in humans. ${ }^{11}{ }^{12}$ In a preclinical pain model of spinal nerve ligation, memantine administered a few days before surgery was shown to prevent the development of neuropathic pain. Molecular biology tests showed a decrease in pTyr $^{1472}$ NR2B concentrations at spinal and supraspinal levels (insula and hippocampus). ${ }^{12}$ The clinical translational study confirmed the beneficial effect of memantine in preventing the development of postmastectomy pain and diminishing chemotherapy-induced pain symptoms and analgesic consumption, with better quality of life for at least 6 months after surgery. ${ }^{11}$

Magnesium is a physiological NMDAR antagonist which blocks the calcium and potassium channels of the receptor, modulating NMDAR activation with very few 
side-effects. ${ }^{13}$ Preclinical and clinical pain studies reported varying curative effects, with satisfactory ${ }^{14-29}$ or more slight results. ${ }^{30-39}$ No study has yet focused on the effect of several weeks oral administration of magnesium starting 2 weeks before surgery on postoperative pain, or more specifically on postoperative pain following breast cancer surgery.

In the present study, magnesium will be administered before surgery in order to evaluate its effect on pain development, cognition, emotion and quality of life during the 3 months following mastectomy. The primary objective is to determine whether magnesium administered for 2 weeks before and 4 weeks after mastectomy may limit pain development at 1 month postmastectomy compared with placebo. The secondary objectives are to assess pain intensity, concomitant analgesic consumption, cognitive-emotional function, quality of life and sleep 1 and 3 months after mastectomy.

\section{METHODS AND ANALYSIS}

\section{Study setting}

A randomised placebo-controlled double-blind clinical trial will be conducted in the Oncology Hospital of Clermont-Ferrand, France, in 100 women undergoing total mastectomy for breast cancer. Three weeks before surgery $\left(\mathrm{D}_{-21}\right)$, patients will meet the medical team, and the physician will explain the protocol and study objectives and the various questionnaires and tests; the patient will then be able provide a written informed consent. As appropriate, sufficient time for reflection will be allowed. After giving informed consent, the women will rate their pain on a numerical rating scale (NRS) and complete the cognition, emotion, quality of life and sleep questionnaires. A blood test will be performed for magnesium and creatinine dosage, and participants will be randomised between two parallel groups: magnesium $(n=50)$ and placebo $(n=50)$. Patients will be contacted 2 weeks before surgery with a reminder to start treatment. Magnesium or placebo (lactose) will be taken orally for 6 weeks, starting 2 weeks before surgery. Magnesium will be given at a dose of $100 \mathrm{mg} /$ day (2 tablets of $50 \mathrm{mg} /$ day once daily). Endpoints will be reassessed at 1 (M1) and 3 months (M3) postmastectomy. Patients will be contacted weekly by phone to maintain good compliance and screen for any adverse events. Finally, patients will have a diary for recording drug intake and adverse events, daily for 3 months starting on the day of surgery $\left(\mathrm{D}_{0}\right)$. Detailed information on the study is summarised in figure 1 .

\section{Inclusion criteria}

1. Age $\geq 18$ years.

2. Diagnosis of breast cancer with scheduled total mastectomy with or without axillary dissection.

3. No change in treatment or diet.

4. Patient able to understand and agreeing to follow the study protocol.

\section{Exclusion criteria}

1. Any contraindications for magnesium: hypersensitivity to magnesium chloride or to any of the excipients.

2. Magnesaemia $>1.05 \mathrm{mmol} / \mathrm{L}$.

3. Severe kidney failure and renal clearance $<30 \mathrm{~mL} / \mathrm{min}$.

4. Alcohol addiction, as diagnosed by the investigator.

5. Type 1 or 2 diabetes.

6. Treatment with quinidine or L-Dopa.

7. Childbearing age with no effective contraceptive method, pregnancy or lactation.

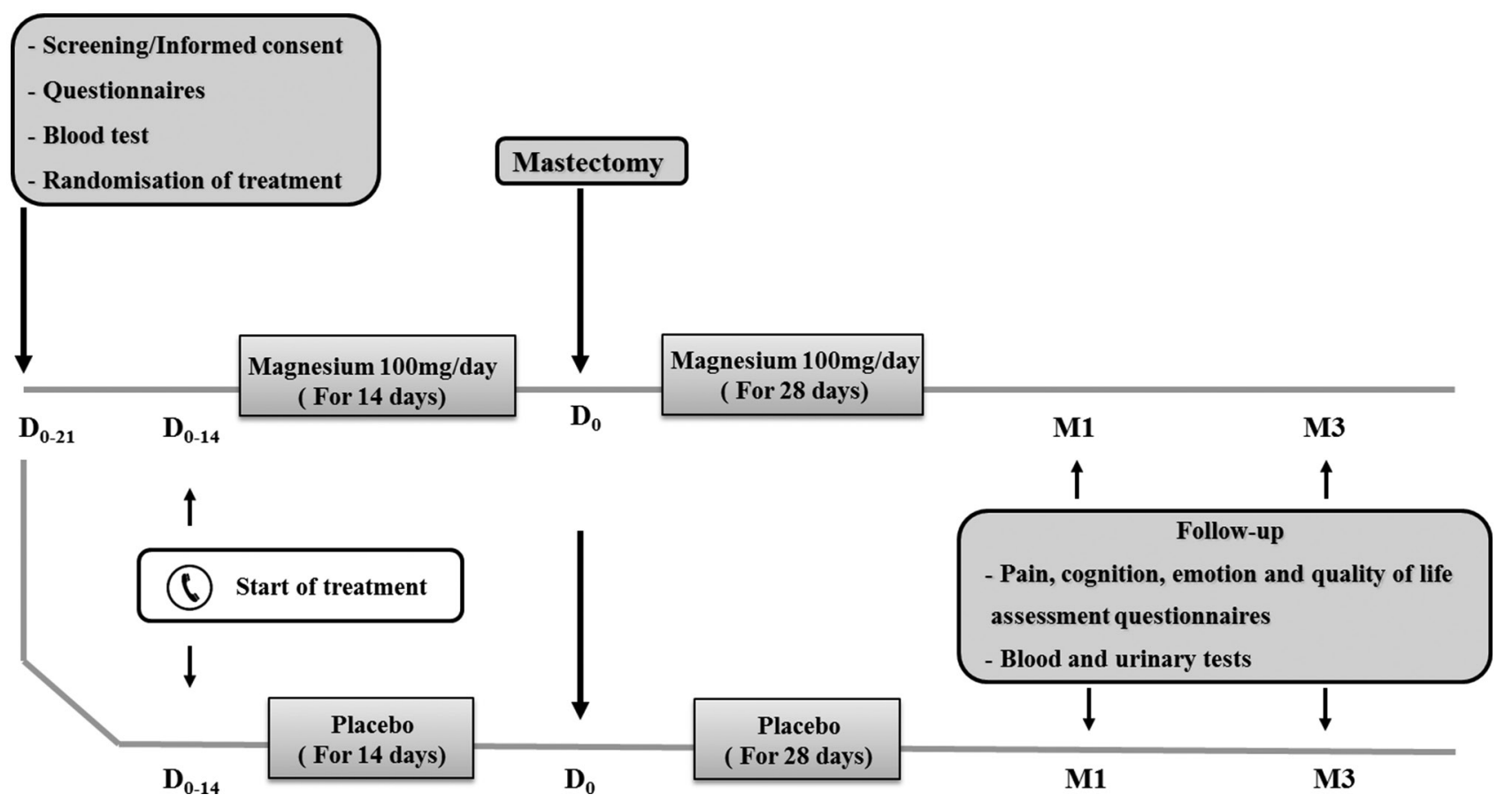

Figure 1 Study design. 
8. Enrolment in another clinical trial.

9. Inability to comply with protocol requirements.

\section{Patient and public involvement}

This study will take place in women suffering from breast cancer and the aim is to determine if magnesium administered before mastectomy may limit pain development at 1 month postsurgery. Patients will be informed about the interest of improved knowledge for the prevention of pain postsurgery and will be asked to collaborate at their best in the study. Patients will not be involved in the recruitment and conduct of the study. Results will be presented when published to the participants in an annual meeting. The burden of the intervention will be assessed by the patients at the end of the study in order to evaluate any potential gaps in the trial.

\section{Intervention}

Treatment group

The treatment group will receive magnesium for 6 weeks, starting 2 weeks before surgery: $100 \mathrm{mg}$ /day (two $50 \mathrm{mg}$ tablets in a single dose) of low-dose continuous-release magnesium (ChronoMag, FJ Life Sciences), stored in opaque white bottles so as to maintain double-blinding.

\section{Control group}

Control patients will receive 2 tablets of placebo (lactose), in a single dose once daily, for 6 weeks, starting 2 weeks before surgery.

Magnesium and packaging will be provided by FJ Life Sciences. The placebo will be prepared, packaged and released in the hospital's central pharmacy by a single qualified person, according to good manufacturing principles. The number of tablets in each container will be checked and recounted at end of treatment by two persons totally independent of the protocol.

\section{Outcome evaluation}

The primary endpoint will be mean pain intensity on NRS in the magnesium and placebo groups over the 5 days preceding the 1-month postsurgery visit. The scale ranges from 0 (no pain) to 10 (maximum tolerable pain).

The following secondary endpoints will be evaluated at the M1 and M3 screening visit: (1) pain, on NRS and McGill pain questionnaire ${ }^{40} ;(2)$ neuropathic pain questionnaires: Neuropathic Pain Questionnaire in 4 Questions (DN4) and Neuropathic Pain Symptom Inventory (NPSI) ${ }^{41} 42$; (3) cognition, on Trail-Making Test (TMT) and Functional Assessment of Cancer Therapy-Cognitive Function (FACT-COG) ${ }^{4344}$; (4) anxiety and depression, on the Depression-Anxiety-Stress Scale (DASS) ${ }^{45}$; (5) quality of life, on the European Organization for Research and Treatment of Cancer Quality of Life Core 30-item and the Patient Global Impression of Change questionnaires; and (6) sleep, on the Pittsburgh Sleep Quality Index. ${ }^{46-48}$ At the inclusion, M1 and M3 visits, blood and urine concentrations of magnesium will be measured. An example of data for one patient is reported in table 1.
McGill Pain Questionnaire

This questionnaire qualifies pain experience during the previous 48 hours. It has 58 qualifiers divided into 16 items (A-P). Each qualifier is rated from 0 to 4 , where $0=$ absent, $1=$ low, $2=$ moderate, $3=$ strong, $4=$ =ery strong. The score is divided into two subclasses: sensory (items A-I) and emotional (items J-P). ${ }^{40}$

\section{Neuropathic Pain Questionnaire in 4 Questions}

DN4 is a clinical tool for the diagnosis of neuropathic pain. This questionnaire has four questions divided into 10 items related to the interview (ie, symptoms) and to the sensory examination (ie, signs). The investigator asks and examines the patient and notes a response 'no' or 'yes' for each item: 'yes' is scored as ' 1 ' and 'no' is scored as ' 0 '. The sum of scores gives the total score of the patient $(/ 10)$. DN4 is considered positive for scores $\geq 4 / 10{ }^{41}$

\section{Neuropathic Pain Symptom Inventory}

NPSI is a self-administered questionnaire comprising 10 pain descriptors. Intensity is rated on 0-10 numerical scales, and two temporal items are designed to assess ongoing spontaneous pain duration and the number of pain paroxysms over 24 hours. This questionnaire discriminates five distinct clinically relevant dimensions: spontaneous burning pain, spontaneous deep pain, paroxysmal pain, evoked pain and paresthesia/dysesthesia. ${ }^{42}$

\section{Trail-Making Test}

This non-verbal cognitive test assesses ability of speed, executive function, attention, concentration and visual perceptual speed. In part A, the patient has to draw lines connecting circles randomly numbered from 1 to 25 , and the patient must connect with lines the numbers in ascending order (1-2-3-4, etc). In part B, the circles are labelled with numbers from 1 to 13 and letters from A to $\mathrm{L}$, and the patient has to draw lines connecting the circles, but alternating numbers and letters $(1 \mathrm{~A}-2 \mathrm{~B}-3 \mathrm{C}$, etc). The patient has to connect the circles as quickly as possible in both parts of the test, without lifting pen from paper. TMT B additionally provides an estimate of mental flexibility. ${ }^{43}$

\section{Functional Assessment of Cancer Therapy-Cognitive Function}

This self-reported questionnaire has been validated in patients with cancer and assesses cognitive impairment and its impact on quality of life. It comprises 37 items, assessing memory, attention, concentration, language and thinking abilities. Items are rated on a 5-point Likert scale. The FACT-COG takes into consideration the functional implications of cognitive impairment, deficits observed by others, changes in cognitive function over time and impact on quality of life. ${ }^{44}$

\section{Depression-Anxiety-Stress Scale}

DASS is a 42-item self-reported instrument designed to measure the three related negative emotional states of depression, anxiety and tension/stress. The DASS-depression scale focuses on reports of low mood, motivation 
Table 1 Summary of assessments

\begin{tabular}{|c|c|c|c|c|c|}
\hline \multirow[b]{3}{*}{ Time point } & \multicolumn{5}{|c|}{ Study period } \\
\hline & \multirow{2}{*}{$\begin{array}{l}\text { Enrolment } \\
\text { D0־21 }\end{array}$} & \multirow{2}{*}{$\begin{array}{l}\text { Allocation } \\
\text { D0-14 }\end{array}$} & \multicolumn{2}{|c|}{ Postallocation } & \multirow{2}{*}{$\begin{array}{l}\text { Close`out } \\
\text { M3 }\end{array}$} \\
\hline & & & D0 & M1 & \\
\hline \multicolumn{6}{|l|}{ Enrolment } \\
\hline Eligibility screen & $\mathrm{x}$ & & & & \\
\hline Informed consent & $x$ & & & & \\
\hline Allocation & & $\mathrm{x}$ & & & \\
\hline \multicolumn{6}{|l|}{ Interventions } \\
\hline Start of treatment & & $\mathrm{x}$ & & & \\
\hline End of treatment & & & & $\mathrm{x}$ & \\
\hline Surgery & & & $\mathrm{x}$ & & \\
\hline Blood test & $x$ & & & $\mathrm{x}$ & $\mathrm{x}$ \\
\hline Urine test & & & & $\mathrm{x}$ & $\mathrm{x}$ \\
\hline Delivery of pain diary & & & $\mathrm{x}$ & & \\
\hline \multicolumn{6}{|l|}{ Assessments } \\
\hline Numeric Rating Scale & $\mathrm{x}$ & & & $\mathrm{x}$ & $\mathrm{x}$ \\
\hline $\begin{array}{l}\text { Neuropathic Pain Questionnaire in } 4 \text { Questions } \\
\text { Neuropathic Pain Symptom Inventory }\end{array}$ & $\mathrm{x}$ & & & $\mathrm{x}$ & $\mathrm{x}$ \\
\hline McGill Pain Questionnaire & $\mathrm{x}$ & & & $x$ & $\mathrm{x}$ \\
\hline Trail'Making Test & $\mathrm{x}$ & & & $\mathrm{x}$ & $\mathrm{x}$ \\
\hline $\begin{array}{l}\text { Functional Assessment of } \\
\text { Cancer Therapy }{ }^{-} \text {Cognitive Function }\end{array}$ & $x$ & & & $x$ & $x$ \\
\hline EORTC QLQ־C30 & $\mathrm{X}$ & & & $\mathrm{X}$ & $\mathrm{x}$ \\
\hline Depression Anxiety Stress Scale & $x$ & & & $\mathrm{x}$ & $x$ \\
\hline Pittsburgh Sleep Quality Index & $\mathrm{x}$ & & & $\mathrm{x}$ & $\mathrm{x}$ \\
\hline Patient Global Impression of Change & $x$ & & & $\mathrm{x}$ & $x$ \\
\hline Concomitant analgesic treatments & $\mathrm{x}$ & $\mathrm{x}$ & $\mathrm{x}$ & $x$ & $\mathrm{x}$ \\
\hline Adverse events & & $\mathrm{x}$ & $\mathrm{x}$ & $\mathrm{x}$ & $\mathrm{x}$ \\
\hline
\end{tabular}

EORTC QLQ C30, European Organization for Research and Treatment of Cancer Quality of Life Core 30-item.

and self-esteem, DASS-anxiety on physiological arousal, perceived panic and fear, and DASS-stress on tension and irritability. The respondent indicates, on a 4-point scale, the extent to which each of the 42 statements applied over the previous week. A printed overlay is used to obtain total scores for each subscale. Higher scores on each subscale indicate increasing severity of depression, anxiety or stress. ${ }^{45}$

European Organization for Research and Treatment of Cancer Quality of Life Questionnaire Core 30 items

This questionnaire assesses the quality of life of patients with cancer. It is divided into nine subscales, consisting of several items: five subscales measuring functional status (physical, role, social, emotional, cognitive), three subscales measuring symptoms (fatigue, pain, nausea and vomiting) and a global subscale of quality of life and health. Finally, six items on isolated symptoms cover cancer symptoms and frequent side effects of cancer therapies. $^{46}$
Patient Global Impression of Change

This is a 7-point self-reported numerical scale assessing what changes in condition following treatment meant to the patient. ${ }^{47}$

\section{Pittsburgh Sleep Quality Index}

This questionnaire consists of 19 items and is used to measure sleep quality. It comprises seven domains: subjective sleep quality, sleep latency, sleep duration, habitual sleep efficiency, sleep disturbances, use of sleep medication and daytime dysfunction. ${ }^{48}$

\section{Recruitment and randomisation}

Three weeks before mastectomy, when the informed consent form has been signed, blood samples will be taken for magnesium and creatinine concentrations, and questionnaires will be delivered. Patients will then be randomised between the magnesium $(n=50)$ and placebo groups $(n=50)$, by a person totally independent of the study, following a predetermined randomisation list 
generated using random blocks. Treatments (magnesium and placebo) will be packed in similar opaque bottles with identically designed labels indicating batch number, expiry date and sponsor code, with no indication of the name of the drug. To maintain blinding, the physician assessing pain will not be able to guess allocation at any time and will not meet the patient again in the course of the trial.

\section{Sample size calculation}

Sample size estimation has been performed using Stata software V.13 (StataCorp) with command sampsi based on usual sample size estimation. ${ }^{49}$ Considering the literature, the prevalence of postmastectomy pain is $20 \% .^{50}$ However, these data may vary depending on demographic, psychological and medical/surgical factors ${ }^{52}$ and will be taken into consideration in this study. The number of subjects required is 100 patients with breast cancer undergoing total mastectomy (50 in each group). The minimum $\delta$ difference in numerical pain scale between magnesium and placebo groups at M1 is estimated at 1.0 and $\sigma$ standard deviation at 1.5 with $\alpha=0.05$ two-sided type I error and $\beta=0.10$.

\section{Statistical analysis}

Statistical analyses will be performed with Stata software V.13. Concerning the primary objective, comparison between the randomised groups will be performed using the Student's t-test or the Mann-Whitney U test (if the conditions for validity of the Student's t-test are not respected, normality verified by Shapiro-Wilk and homoscedasticity by Fisher-Snedecor test). If a high correlation between baseline and follow-up scores is highlighted, an analysis of covariance with the baseline average NRS as a covariate will be proposed as multivariable analysis, as proposed by Vickers and Altman. ${ }^{53}$ This analysis can thus be expanded to include additional prognostic variables. The confounding factors likely to influence the primary endpoint (paravertebral block, breast reconstruction with latissimus dorsi muscle flap, axillary dissection) will be taking into account in multivariate regression analysis. Concerning anaesthesia, it is generally standardised and the authorised treatment will be noted. There will be a systematic adjustment for the main analysis. The analysis of repeated data (at the inclusion, M1 and M3) will be carried out by mixed models which allow to consider, on the one hand, time, group and their interaction time $x$ group as fixed effects and on the other hand, the within and between subject variability. A sensitivity analysis will be considered to measure the impact of missing data and to assess the problem caused by missing longitudinal data at M3. A sensitivity analysis will be performed to measure the impact of missing data and to assess the problem caused by missing longitudinal data at M3. The nature of missing data will be studied (missing at random or not). According to this, the most appropriate approach to the imputation of missing data will be proposed: multiple imputation, maximum bias (last observation carried forward vs baseline observation carried forward) or estimation proposed by Verbeke and Molenberghs for repeated data'.

\section{Data handling and record keeping}

All original records, such as consent forms, case report forms, questionnaires and pain diaries, will be archived at trial sites for 15 years. The database file will be anonymised and likewise archived for 15 years. Monitoring will be performed by a clinical research associate independent of the study. The monitored case report forms will then be transferred to the Data Management Center (CIC-Inserm 1405, Clermont-Ferrand).

\section{Study duration}

Treatment duration will be 42 days. Total participation duration per patient will be 14 weeks. The protocol will include four visits $\left(\mathrm{D}_{-21} / \mathrm{D}_{0} / \mathrm{M} 1 / \mathrm{M} 3\right)$, with a systematic period of hospital admission for surgery. Treatment will be taken daily, starting 2 weeks before surgery and maintained 4 weeks after. Recruitment will start in June 2018. The total duration of the study is estimated at 3 years.

\section{Ethics and dissemination}

Each patient meeting the inclusion criteria will sign a consent form after receiving oral and written information. After agreement between all investigators, data will be disclosed and results will be communicated in various congresses and published in international publications.

\section{DISCUSSION}

Following successful results obtained with prophylactic memantine against onset of neuropathic pain, ${ }^{11} 12$ this study aims at assessing magnesium treatment to prevent neuropathic pain induced by mastectomy, with a similar protocol. In breast cancer surgery, clinical studies using magnesium have focused so far on the qualitative and emotional aspects rather than the actual intensity of pain. ${ }^{55}$ Magnesium has shown discrepant results in alleviating neuropathic pain. ${ }^{18-39}$ It has been shown to modulate the limbic system via NMDAR, and these brain areas are known to be involved in emotion and pain. ${ }^{56}$ It is therefore essential to evaluate magnesium's effect on pain and concomitantly on cognitive-emotional aspects and sleep. Magnesium deprivation may affect cognition and sleep quality. Preclinical findings showed that increased brain magnesium levels enhanced both shortterm synaptic facilitation and long-term potentiation and improved learning and memory functions. ${ }^{57}$ In humans, patients with pre-eclampsia receiving magnesium showed better attention and working memory performance than controls, both before and after delivery. ${ }^{58}$ Furthermore, a review reported the relationship between low magnesium level and stress or cognitive difficulties such as lack of concentration and difficulties in learning. ${ }^{59}$ Concerning the impact of magnesium on sleep, a placebo-controlled randomised cross-over study performed in 
12 elderly participants showed that magnesium supplementation significantly reversed electroencephalogram changes, including decreased slow-wave sleep, that may occur during ageing. ${ }^{60}$ Furthermore, a double-blind trial reported that intraoperative infusion of magnesium significantly improved quality of sleep during the postoperative period, without any side effects. ${ }^{28}$

Magnesium is an abundant mineral, naturally present in food, and is available as a dietary supplement that is appreciated by patients. ${ }^{61}$ It can be obtained without prescription and has a favourable risk-benefit ratio, with few side effects. ${ }^{61}$ It is also known to regulate diverse biochemical reactions in the body, and is required for energy production, oxidative phosphorylation and glycolysis. It also plays a role in the active transport of calcium and potassium ions across cell membranes, a process that is important for nerve impulse conduction, muscle contraction and normal heart rhythm. ${ }^{62}{ }^{63}$ Low blood levels of magnesium were shown to be associated with a number of pathologies, including type 2 diabetes and cardiovascular disease. ${ }^{64}$ Oral magnesium supplementation is usually well tolerated and gastrointestinal side effects such as nausea, vomiting and diarrhoea are usually minor. $^{6465}$

The pharmaceutical form used in this trial provides magnesium chloride, a circulating form of magnesium with gradual constant release of low-dose magnesium along the gastrointestinal tract. A recent clinical study (NCT01935570) showed that a dose of $100 \mathrm{mg}$ daily ensures optimal absorption over a 24-hour period. Furthermore, this form of magnesium does not induce intestinal side effects and is easy to use, with once-a-day intake.

In conclusion, if magnesium given before and after mastectomy proves its efficacy in preventing neuropathic pain, it could provide an excellent prophylactic strategy against postmastectomy pain symptoms, maintain quality of life and cognitive function and limit the comorbidities that accompany breast cancer.

\section{Author affiliations}

${ }^{1} \mathrm{CHU}$ Clermont-Ferrand, Inserm CIC Inserm 1405, Centre de Pharmacologie Clinique, Clermont-Ferrand, France

${ }^{2} \mathrm{CHU}$ Clermont-Ferrand, Centre Jean Perrin, Centre de Lutte contre le Cancer, Clermont-Ferrand, Auvergne, France

${ }^{3} \mathrm{CHU}$ de Clermont-Ferrand, Délégation Recherche Clinique \& Innovation - Villa annexe IFSI, Clermont-Ferrand, France

${ }^{4}$ Université Clermont Auvergne, Laboratoire de Pharmacologie, Facultés de Médecine/Pharmacie, Clermont-Ferrand, France

${ }^{5}$ Inserm, U1107 Neuro-Dol, Pharmacologie Fondamentale et Clinique de la Douleur, Clermont-Ferrand, France

Contributors GP is the overall principal study investigator; she participated in the study design and contributed to drawing up the study protocol and drafting and editing of this manuscript. DJ, CV and BP all participated in the study design. BP contributed to drawing up the study protocol, carried out all statistical calculations and wrote the Statistics section in the study protocol. He contributed with GP and VM to drafting and editing the manuscript. All authors read and approved the final manuscript.

Funding University Hospital Clermont-Ferrand, France.

Competing interests None declared.

\section{Patient consent Obtained.}

Ethics approval The study was approved in December 2016 by the regional ethics committee (2016-A01749-42)

Provenance and peer review Not commissioned; externally peer reviewed.

Open access This is an open access article distributed in accordance with the Creative Commons Attribution Non Commercial (CC BY-NC 4.0) license, which permits others to distribute, remix, adapt, build upon this work non-commercially, and license their derivative works on different terms, provided the original work is properly cited, appropriate credit is given, any changes made indicated, and the use is non-commercial. See: http://creativecommons.org/licenses/by-nc/4.0/.

\section{REFERENCES}

1. Rojas K, Stuckey A. Breast Cancer Epidemiology and Risk Factors. Clin Obstet Gynecol 2016;59:651-72.

2. Labrèze L, Lakdja F, Dixmérias F, et al. Les douleurs chroniques postmastectomie. Douleur et Analgésie 2009;22:30-7.

3. Collins S, Sigtermans MJ, Dahan A, et al. NMDA receptor antagonists for the treatment of neuropathic pain. Pain Med 2010;11:1726-42.

4. Niesters M, Dahan A. Pharmacokinetic and pharmacodynamic considerations for NMDA receptor antagonists in the treatment of chronic neuropathic pain. Expert Opin Drug Metab Toxicol 2012;8:1409-17.

5. Zhou HY, Chen SR, Pan HL. Targeting N-methyl-D-aspartate receptors for treatment of neuropathic pain. Expert Rev Clin Pharmacol 2011;4:379-88.

6. Pickering G. La voie glutamatergique: aspects physiologiques et pharmacologiques du récepteur NMDA. Lett Pharmacol Supplément 2010;23:4-12.

7. Rogawski MA, Wenk GL. The neuropharmacological basis for the use of memantine in the treatment of Alzheimer's disease. CNS Drug Rev 2003;9:275-308.

8. Vandenbossche S, Fery P, Razavi D. [Cognitive impairments and breast cancer: a critical review of the literature]. Bull Cancer 2009;96:239-48.

9. Bell RF. Ketamine for chronic non-cancer pain. Pain 2009;141:210-4.

10. Ehret GB, Daali Y, Chabert J, et al. Influence of CYP2D6 activity on pre-emptive analgesia by the $\mathrm{N}$-methyl-D-aspartate antagonist dextromethorphan in a randomized controlled trial of acute pain. Pain Physician 2013;16:45-56.

11. Morel V, Joly D, Villatte $\mathrm{C}$, et al. memantine before mastectomy prevents post-surgery pain: a randomized, blinded clinical trial in surgical patients. PLoS One 2016;11:e0152741.

12. Morel V, Etienne M, Wattiez AS, et al. Memantine, a promising drug for the prevention of neuropathic pain in rat. Eur $\mathrm{J}$ Pharmacol 2013;721:382-90.

13. Woolf CJ, Thompson SW. The induction and maintenance of central sensitization is dependent on N-methyl-D-aspartic acid receptor activation; implications for the treatment of post-injury pain hypersensitivity states. Pain 1991;44:293-9.

14. Begon S, Pickering G, Eschalier A, et al. Magnesium and MK-801 have a similar effect in two experimental models of neuropathic pain. Brain Res 2000;887:436-9.

15. Begon S, Pickering G, Eschalier A, et al. Magnesium increases morphine analgesic effect in different experimental models of pain. Anesthesiology 2002;96:627-32.

16. Begon S, Pickering G, Eschalier A, et al. Role of spinal NMDA receptors, protein kinase $\mathrm{C}$ and nitric oxide synthase in the hyperalgesia induced by magnesium deficiency in rats. $\mathrm{Br} J$ Pharmacol 2001;134:1227-36.

17. Hasanein P, Parviz M, Keshavarz M, et al. Oral magnesium administration prevents thermal hyperalgesia induced by diabetes in rats. Diabetes Res Clin Pract 2006;73:17-22.

18. Apan A, Buyukkocak U, Ozcan S, et al. Postoperative magnesium sulphate infusion reduces analgesic requirements in spinal anaesthesia. Eur J Anaesthesiol 2004;21:766-9.

19. Kara H, Sahin N, Ulusan V, et al. Magnesium infusion reduces perioperative pain. Eur J Anaesthesiol 2002;19:52-6.

20. Levaux C, Bonhomme V, Dewandre PY, et al. Effect of intra-operative magnesium sulphate on pain relief and patient comfort after major lumbar orthopaedic surgery. Anaesthesia 2003;58:131-5.

21. Shah PN, Dhengle Y. Magnesium sulfate for postoperative analgesia after surgery under spinal anesthesia. Acta Anaesthesiol Taiwan 2016;54:62-4.

22. Steinlechner B, Dworschak M, Birkenberg B, et al. Magnesium moderately decreases remifentanil dosage required for pain management after cardiac surgery. Br J Anaesth 2006;96:444-9. 
23. Brill S, Sedgwick PM, Hamann W, et al. Efficacy of intravenous magnesium in neuropathic pain. Br J Anaesth 2002;89:711-4.

24. Crosby V, Wilcock A, Corcoran R. The safety and efficacy of a single dose $(500 \mathrm{mg}$ or $1 \mathrm{~g}$ ) of intravenous magnesium sulfate in neuropathic pain poorly responsive to strong opioid analgesics in patients with cancer. J Pain Symptom Manage 2000;19:35-9.

25. Jaitly $\mathrm{V}$. Efficacy of intravenous magnesium in neuropathic pain. $\mathrm{Br} J$ Anaesth 2003;91:302.

26. Peikert A, Wilimzig C, Köhne-Volland R. Prophylaxis of migraine with oral magnesium: results from a prospective, multi-center, placebo-controlled and double-blind randomized study. Cephalalgia 1996;16:257-63.

27. Shechter M, Bairey Merz CN, Stuehlinger HG, et al. Effects of oral magnesium therapy on exercise tolerance, exercise-induced chest pain, and quality of life in patients with coronary artery disease. Am J Cardiol 2003;91:517-21.

28. Bhatia A, Kashyap L, Pawar DK, et al. Effect of intraoperative magnesium infusion on perioperative analgesia in open cholecystectomy. J Clin Anesth 2004;16:262-5.

29. Tramer MR, Schneider J, Marti RA, et al. Role of magnesium sulfate in postoperative analgesia. Anesthesiology 1996;84:340-7.

30. Pfaffenrath V, Wessely P, Meyer C, et al. Magnesium in the prophylaxis of migraine-a double-blind placebo-controlled study. Cephalalgia 1996;16:436-40.

31. Wang F, Van Den Eeden SK, Ackerson LM, et al. Oral magnesium oxide prophylaxis of frequent migrainous headache in children: a randomized, double-blind, placebo-controlled trial. Headache 2003;43:601-10.

32. Baaklini LG, Arruda GV, Sakata RK. Assessment of the Analgesic Effect of Magnesium and Morphine in Combination in Patients With Cancer Pain: A Comparative Randomized Double-Blind Study. Am J Hosp Palliat Care 2017;34:353-7.

33. Ko SH, Lim HR, Kim DC, et al. Magnesium sulfate does not reduce postoperative analgesic requirements. Anesthesiology 2001;95:640-6.

34. O'Flaherty JE, Lin CX. Does ketamine or magnesium affect posttonsillectomy pain in children? Paediatr Anaesth 2003;13:413-21.

35. Tramèr MR, Glynn CJ. An evaluation of a single dose of magnesium to supplement analgesia after ambulatory surgery: randomized controlled trial. Anesth Analg 2007;104:1374-9.

36. Pickering G, Morel V, Simen E, et al. Oral magnesium treatment in patients with neuropathic pain: a randomized clinical trial. Magnes Res 2011;24:28-35.

37. Zarauza R, Sáez-Fernández AN, Iribarren MJ, et al. A comparative study with oral nifedipine, intravenous nimodipine, and magnesium sulfate in postoperative analgesia. Anesth Analg 2000;91:938-43.

38. Felsby S, Nielsen J, Arendt-Nielsen L, et al. NMDA receptor blockade in chronic neuropathic pain: a comparison of ketamine and magnesium chloride. Pain 1996;64:283-91.

39. Mikkelsen S, Dirks J, Fabricius P, et al. Effect of intravenous magnesium on pain and secondary hyperalgesia associated with the heat/capsaicin sensitization model in healthy volunteers. $\mathrm{Br} J$ Anaesth 2001;86:871-3.

40. Boureau F, Luu M, Doubrère JF. Comparative study of the validity of four French McGill Pain Questionnaire (MPQ) versions. Pain 1992;50:59-65.

41. Bouhassira $\mathrm{D}$, Attal $\mathrm{N}$, Alchaar $\mathrm{H}$, et al. Comparison of pain syndromes associated with nervous or somatic lesions and development of a new neuropathic pain diagnostic questionnaire (DN4). Pain 2005;114:29-36.

42. Bouhassira D, Attal N, Fermanian J, et al. Development and validation of the Neuropathic Pain Symptom Inventory. Pain 2004;108:248-57.
43. Tombaugh TN. Trail Making Test A and B: normative data stratified by age and education. Arch Clin Neuropsychol 2004;19:203-14.

44. Wagner L, Sweet J, Butt Z, et al. Measuring patient self-reported cognitive function: development of the functional assessment of cancer therapy-cognitive function instrument. J Support Oncol 2009; 7:W32-9.

45. Lovibond SH, Lovibond PF. Manual for the depression anxiety and stress scales. 2nd edn: Psychology Foundation.

46. Aaronson NK, Ahmedzai S, Bergman B, et al. The European Organization for Research and Treatment of Cancer QLQ-C30: a quality-of-life instrument for use in international clinical trials in oncology. J Natl Cancer Inst 1993;85:365-76.

47. Hurst $\mathrm{H}$, Bolton J. Assessing the clinical significance of change scores recorded on subjective outcome measures. $J$ Manipulative Physiol Ther 2004;27:26-35.

48. Buysse DJ, Reynolds CF, Monk TH, et al. The pittsburgh sleep quality index: a new instrument for psychiatric practice and research. Psychiatry Res 1989;28:193-213.

49. Machin D, Campbell MJ, Tan SB, et al. Sample size tables for clinical studies. 3rd edn. Chichester: Wiley-Blackwell, 2009.

50. Stevens PE, Dibble SL, Miaskowski C. Prevalence, characteristics, and impact of postmastectomy pain syndrome: an investigation of women's experiences. Pain 1995;61:61-8.

51. Smith WC, Bourne D, Squair J, et al. A retrospective cohort study of post mastectomy pain syndrome. Pain 1999;83:91-5.

52. Schreiber KL, Kehlet H, Belfer I, et al. Predicting, preventing and managing persistent pain after breast cancer surgery: the importance of psychosocial factors. Pain Manag 2014;4:445-59.

53. Vickers AJ, Altman DG. Statistics notes: analysing controlled trials with baseline and follow up measurements. BMJ 2001;323:1123-4.

54. Verbeke G, Fieuws S, Molenberghs G, et al. The analysis of multivariate longitudinal data: a review. Stat Methods Med Res 2014;23:42-59.

55. Tao MH, Dai Q, Millen AE, et al. Associations of intakes of magnesium and calcium and survival among women with breast cancer: results from Western New York Exposures and Breast Cancer (WEB) Study. Am J Cancer Res 2016;6:105-13.

56. Bardgett ME, Schultheis PJ, McGill DL, et al. Magnesium deficiency impairs fear conditioning in mice. Brain Res 2005;1038:100-6.

57. Slutsky I, Abumaria N, Wu LJ, et al. Enhancement of learning and memory by elevating brain magnesium. Neuron 2010;65:165-77.

58. Rana S, Lindheimer M, Hibbard J, et al. Neuropsychological performance in normal pregnancy and preeclampsia. Am J Obstet Gynecol 2006;195:186-91.

59. Moncayo R, Ortner K. Multifactorial determinants of cognition Thyroid function is not the only one. BBA Clin 2015;3:289-98.

60. Held K, Antonijevic IA, Künzel H, et al. Oral $\mathrm{Mg}(2+)$ supplementation reverses age-related neuroendocrine and sleep EEG changes in humans. Pharmacopsychiatry 2002;35:135-43.

61. National Institutes of Health Office of Dietary Supplements. Magnesium. http://ods.od.nih.gov/factsheets/magnesium.asp (accessed 12 Jan 2009)

62. Newhouse IJ, Finstad EW. The effects of magnesium supplementation on exercise performance. Clin J Sport Med 2000;10:195-200.

63. Chubanov V, Gudermann T, Schlingmann KP. Essential role for TRPM6 in epithelial magnesium transport and body magnesium homeostasis. Pflugers Arch 2005;451:228-34.

64. Guerrera MP, Volpe SL, Mao JJ. Therapeutic uses of magnesium. Am Fam Physician 2009;80:157-62.

65. McKevoy GK, ed. AHFS drug information. Bethesda, MD: American Society of Health-System Pharmacists, 1998. 